\title{
The coupled criterion for description of fatigue fracture. Material embrittlement in pre-fracture zone
}

\author{
Vladimir M. Kornev ${ }^{*}$
}

Lavrentyev Insti subareas tute of Hydrodynamics SB RAS, Novosibirsk, 630090, Russia

\begin{abstract}
Step-wise extension of a crack in quasi-brittle materials under low-cycle loading conditions is considered. Both steady and unsteady loadings in pulsating loading mode are studied. It is proposed to use quasi-brittle fracture diagrams for bodies under cyclic loading conditions. When diagrams are plotted, both necessary and sufficient fracture criteria by Neuber-Novozhilov are used. A specific implementation is made on the base of the Leonov-Panasyuk-Dugdale model for the mode I cracks when the pre-fracture zone width coincides with the plasticity zone width near the crack tip. The condition of a step-wise crack tip extension has been derived. A crack extends only in the embrittled material of the pre-fracture zone. The number of cycles between jumps of the crack tip is calculated by the Coffin equation, when damage accumulation in material in the pre-fracture zone is taken into account. Critical fracture parameters under low-cyclic loading conditions have been obtained in a closed form. Estimates of the average rate of crack tip advance for a loading cycle at step-wise crack extension and $S-N$ curves have been obtained.
\end{abstract}

\section{Introduction}

In order to obtain estimates for the number of cycles, the diagram of quasi-brittle fracture under low-cycle fatigue is used $[1,2]$.

The problems to be settled in this work are as follows: $i$ ) to derive relations for critical stresses above which the growth of this macrocrack takes place in specimens having a short macro-crack; ii) in the plane stresses vesus crack length plotting a subarea where damage accumulation occurs after single loading; iii) description of the process of step-wise propagation of short macro-cracks under fatigue when material embrittlement in the pre-fracture zone takes place; $i v$ ) selection of parametrs for the proposed model acording to the resuls of laboratory tests.

\section{Diagrams of quasi-brittle fracture under single loading}

Apply the simplest approximation of a $\sigma-\varepsilon$ diagram for elastic-plastic material when the diagram is approximated by a double-link straight line. The parameters of this approximation are as follows: $E$ is the modulus of elasticity, $\sigma_{Y}$ is the yield strength of material, and $\sigma_{Y}$ is constant stress acting in accordance with the modified Leonov-Panasyuk-Dugdale model, $\varepsilon_{0}$ is the maximum elastic material strain $\left(\sigma_{Y}=E \varepsilon_{0}\right), \varepsilon_{1}$ is the maximum material strain. Let $r$ be the effective diameter of fracture structures. The Neuber-Novozhilov approach $[3,4]$ makes it possible to use solutions having a singular function for structured media.

Now consider an internal I mode crack. Assume that an internal plane I mode crack extends rectilinearly. In addition to the internal rectilinear crack-cut of length $2 l_{0}$, model crack-cuts of length $2 l=2 l_{0}+2 \Delta$, each of prefracture zones $\Delta$ being located on the continuation of a real crack ( $2 l$ and $\Delta$ are lengths of model crack and pre-fracture zones) are introduced into consideration. The problem of fatigue fracture has two linear scales: if a grain diameter is defined by a material structure, then the second linear size is governed by the system itself. Under low-cycle fatigue conditions, the second linear scales serve as pre-fracture zone lengths $\Delta$, which change in accordance with changing $i$ ) the length of a real step-wise extending crack, and ii) the intensity of loading conditions. Emphasize that under single loading conditions, the critical pre-fracture zone length $\Delta^{*}$ is a completely definite parameter $[1,2]$ and $2 l^{*}=2 l_{0}+2 \Delta^{*}$ is the critical macro-crack length, that is $\Delta^{*} / l_{0}=1$ for quasi-brittle materials.

When diagrams of quasi-brittle fracture under conditions of low-cycle loading are plotted in $[1,2,5]$, sufficient fracture criteria are used when the mode I cracks are considered

$$
\frac{1}{r} \int_{0}^{r} \sigma_{y}(x, 0) d x=\sigma_{Y}, 2 v(x, 0)=\delta^{*} .
$$

Here $\sigma_{y}(x, 0)$ are normal stresses on the crack continuation; $O x y$ is the rectangular coordinate system oriented about right crack sides (the coordinate origin coincides with the model crack tip in the modified Leonov-Panasyuk-Dugdale model $\left[\begin{array}{lll}1, & 2,5\end{array}\right]$ ); $2 v=2 v(x, 0)$ is CTOD (crack tip opening displacement, $x<0), \delta^{*}$ is the critical CTOD.

The field of normal stresses $\sigma_{y}(x, 0)$ on the model crack continuation $x>0$ can be represented as a sum of two summands 


$$
\begin{aligned}
\sigma_{y}(x, 0) & \cong K_{\mathrm{I}} /(2 \pi x)^{1 / 2}+O(1), \\
K_{\mathrm{I}} & =K_{\mathrm{I} \infty}+K_{\mathrm{I} \Delta}, K_{\mathrm{I} \infty}>0, K_{\mathrm{I} \Delta}<0,
\end{aligned}
$$

where $K_{\mathrm{I}}=K_{\mathrm{I}}(l, \Delta)$ is the total stress intensity factor (SIF) at the tips of a model crack, $K_{\text {Iळ }}$ is SIF generated by stresses $\sigma_{\infty}$ specified at infinity, $K_{\mathrm{I} \Delta}$ is SIF generated by constant stresses $\sigma_{Y}$. The first and second summands in relation (2) are singular and smooth parts of solution, respectively. The first equality in criterion (1) controls stresses on the model crack continuation after averaging, these stresses being coincident with the yield strength $\sigma_{Y}$, and the second equality in criterion (1) describes real blunting of a crack at its tip. The sufficient fracture criterion (1) simultaneously takes into consideration both strength and deformation fracture criteria at specific points of a pre-fracture zone. Selected specific points of the pre-fracture zone are well-adapted to description of step-wise crack tip advance and accumulation of damages in the pre-fracture zone material under fatigue conditions $[1,2]$.

The simplest analytical representation of the field of stresses $\sigma_{y}(x, 0)$ on the model crack continuation has the following form ( $2 L$ is the plain specimen width)

$$
\begin{aligned}
& \sigma_{y}(x, 0)=\left(K_{\mathrm{I} \infty}+K_{\mathrm{I} \Delta}\right) / \sqrt{2 \pi x}+\sigma_{\text {nom }}= \\
= & \sigma_{\infty} Y(l / L) \sqrt{l / 2 x}+2 \sigma_{Y} \sqrt{\Delta / x} / \pi+\sigma_{\infty} /(1-l / L)
\end{aligned}
$$

Introduce into consideration critical stresses calculated via the necessary fracture criterion $\sigma_{\infty}^{0}$ and sufficient one $\sigma_{\infty}^{*}$ and $\Delta^{*}$. System (1) is simplified when fracture of quasi-brittle materials is studied under conditions of small-scale yielding $\Delta^{*} / l_{0}=1$ [2]. In what follows, dimensionless variables are used ( $\mu$ is Poisson's ratio) $\bar{\sigma}_{\infty}^{*}=\sigma_{\infty}^{*} / \sigma_{Y}, \bar{\sigma}_{\infty}^{0}=\sigma_{\infty}^{0} / \sigma_{Y}, \bar{\Delta}^{*}=\Delta^{*} / r, \bar{l}^{*}=l^{*} / r$, $r=(2 / \pi)\left(K_{\mathrm{Ic}} / \sigma_{Y}\right)^{2}\left(1-A \bar{\varepsilon}_{p}\right)^{2}, \quad \bar{\varepsilon}_{p}=\left(\varepsilon_{1}-\varepsilon_{0}\right) / \varepsilon_{0}$, $\bar{L}=L / r, \quad A=\left[3+2(1-2 \mu)^{2}\right] /\left[8 \pi\left(1-\mu^{2}\right)\right] . \quad$ The approximate system of equations is valid for short macrocracks if $2 \bar{l}^{*} / r \geq 0.1 \div 0.2$.

Fracture diagrams for quasi-brittle materials are pairs of curves $\bar{\sigma}_{\infty}^{0}$ and $\bar{\sigma}_{\infty}^{*}$ Analytical representations of critical fracture parameters for quasi-brittle materials has the form for a plain specimen of the finite width $[1,5]$

$$
\begin{aligned}
\bar{\sigma}_{\infty}^{*} \approx & {\left[1 /\left(1-\bar{l}^{*} / \bar{L}\right)+\left(1-A \bar{\varepsilon}_{p}\right) Y\left(\bar{l}^{*} / \bar{L}\right) \sqrt{2 \bar{l}^{*}}\right]^{-1}, } \\
\bar{\sigma}_{\infty}^{0} \approx & {\left[1 /\left(1-\bar{l}_{0} / \bar{L}\right)+Y\left(\bar{l}_{0} / \bar{L}\right) \sqrt{2 \bar{l}_{0}}\right]^{-1}, } \\
& \bar{\Delta}^{*} / \bar{l}^{*} \approx\left(\pi^{2} / 8\right)\left[A \bar{\varepsilon}_{p} Y\left(\bar{l}^{*} / \bar{L}\right) \bar{\sigma}_{\infty}^{*}\right]^{2} .
\end{aligned}
$$

Expressions (4) make sense if $A \bar{\varepsilon}_{p}<1$.

The finite specimen width effects upon critical fracture curves on the plane "crack length versus internal load".

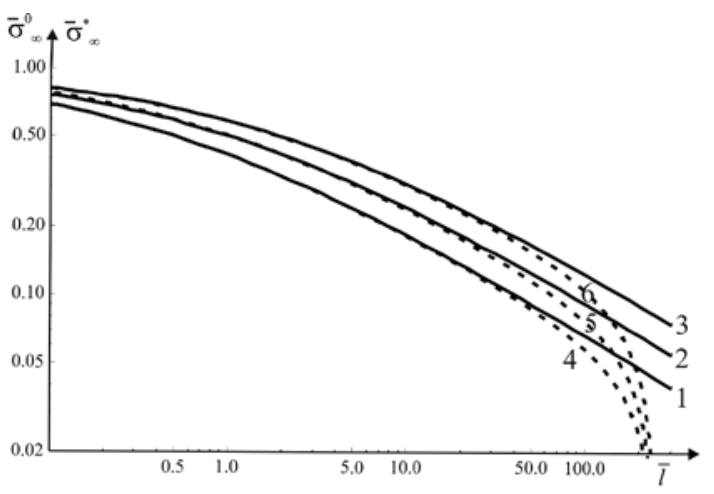

Fig. 1. Fracture diagrams for plain specimens.

The calculation results are given in Fig. 1 in the $\log \log \quad$ coordinates, when $\quad \bar{L}=250$ $(Y(l / L)=\sqrt{\sec (\pi l / 2 L)})$. In this Figure, solid curve 1 and dashed curve 4 are plotted via relation (4) for $\bar{\sigma}_{\infty}^{0}$ at $\bar{L}=\infty$ and $\bar{L}=250$, respectively; solid curves 2 and 3 $(\bar{L}=\infty)$, and dashed lines 5 and $6(\bar{L}=250)$ are plotted via relation (4) for $\bar{\sigma}_{\infty}^{*}$ at $\bar{\varepsilon}_{p}=1.5$ and $\bar{\varepsilon}_{p}=2.5$. Pairs of curves 1-2, 1-3, and 4-5, 4-6 in Fig. 1 are diagrams of quasi-brittle fracture for plain specimens of infinite and finite width, respectively. Under cyclic loading conditions the subareas between curves $\bar{\sigma}_{\infty}^{0}$ and $\bar{\sigma}_{\infty}^{*}$ are areas, in which damage accumulation in pre-fracture zone material takes place.

\section{Step-wise crack propagation}

When the proposed diagram under low-cycle fatigue [1, $2,5]$ is plotted, there is no need to use SIFs. It can be plotted depending on both elastic-plastic material properties and a crack length.

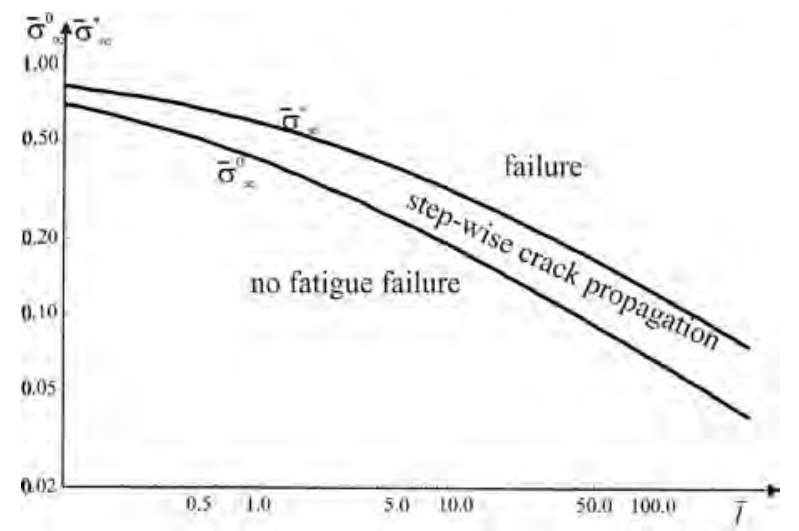

Fig. 2. Diagram of fatigue fracture.

This diagram in Fig. 2 consisting of a pair of curves divides the plane of "crack length versus. external load amplitude" $\left(\bar{l}, \bar{\sigma}_{\infty}\right)$ into three subareas: 1) the subarea where fatigue fracture is not observed is located to the left and lower the curve $\bar{\sigma}_{\infty}^{0} ; 2$ ) the subarea of fracture at single loading is located to the right and above the curve $\bar{\sigma}_{\infty}^{*}$;3) the subarea of step-wise crack 
propagation due to embrittlement of material under cyclic loading conditions is located between curves $\bar{\sigma}_{\infty}^{0}$ and $\bar{\sigma}_{\infty}^{*}$. When repeated loads are applied, the curve $\bar{\sigma}_{\infty}^{*}$ is transformed, embrittlement of the pre-fracture zone material being considered. The proposed diagram has two threshold values $\bar{\sigma}_{\infty}^{0}$ and $\bar{\sigma}_{\infty}^{*}$ in relation (4). loading

Consider limitations typical for one-frequency

$$
\bar{\sigma}_{\infty}^{0}<\bar{\sigma}_{\infty}<\bar{\sigma}_{\infty}^{*} .
$$

Here $\bar{\sigma}_{\infty}$ is amplitude. When limitations (5) are obeyed, the low-cycle fatigue may be implemented. Changes in the subarea of the diagram of quasi-brittle fracture with account for damage accumulation are described for the normal loading regime

$$
\begin{aligned}
& \bar{\sigma}_{\infty}^{0}<\bar{\sigma}_{\infty}<\bar{\sigma}_{\infty}^{*(s)}, 1 \leq s_{j} \leq N_{j}-1, j=1,2, \ldots, j^{*}-1, \\
& \bar{\sigma}_{\infty}^{0}<\bar{\sigma}_{\infty}^{*(s)}<\ldots<\bar{\sigma}_{\infty}^{*(2)}<\bar{\sigma}_{\infty}^{*(1)}=\bar{\sigma}_{\infty}^{*} ; \\
& \bar{\sigma}_{\infty} \geq \bar{\sigma}_{\infty}^{*(s)}, s_{j}=N_{j}, j=1,2, \ldots, j^{*}, \bar{\sigma}_{\infty}^{0}<\bar{\sigma}_{\infty}^{*(s)},
\end{aligned}
$$

when the critical number of loading cycles $N^{*}$ is calculated in such a way

$$
N^{*}=1+\sum_{1}^{j^{*}-1} N_{j}, N_{j} \geq 2, j=1,2, \ldots, j^{*}-1, N_{j^{*}}=1
$$

Here $\sigma_{\infty}^{*(s)}$ is the critical load obtained via the sufficient fracture criterion in $s$-th loading cycle up to $j=1,2, \ldots, j^{*}-1$ crack tip advance, $s_{j}$ is number of cycle between $j-1$ and $j$ crack tip advances, $s_{j}=1$ corresponds to the state of original material after each crack tip advance, $N_{j}$ is the number (group) of cycles between $(j-1)$ and $j$ crack tip advances. For the loading level $\bar{\sigma}_{\infty}$ of a real crack of length $2 l_{j-1}$, equations for specimens of finite width (4) are as follows

$$
\begin{gathered}
\bar{\sigma}_{\infty} \approx\left[1 /\left(1-\bar{l}_{j-1} / \bar{L}\right)+\left(1-A \bar{\varepsilon}_{\infty, j-1}\right) Y\left(\bar{l}_{j-1} / \bar{L}\right) \sqrt{2 \bar{l}_{j-1}}\right]^{-1}, \\
\bar{\Delta}_{j} / \bar{l}_{j-1} \approx\left(\pi^{2} / 8\right)\left[A \bar{\varepsilon}_{\infty, j-1} Y\left(\bar{l}_{j-1} / \bar{L}\right) \bar{\sigma}_{\infty}\right]^{2}, \\
2 \bar{l}_{j}=2 \bar{l}_{j-1}+2 \bar{\Delta}_{j}, j=1,2, \ldots, j^{*} .
\end{gathered}
$$

The number of cycles $N_{j}$ between $j-1$ and $j$ crack tip advances at the loading level $\bar{\sigma}_{\infty}$ is calculated as follows

$$
N_{j}=\left(\bar{\varepsilon}_{p} / \bar{\varepsilon}_{\infty, j-1}\right)^{1 / C}, j=1,2, \ldots, j^{*}-1 .
$$

Here $0.2 \leq C \leq 1$ are Coffin's constants [6], numerical values of which depend on material properties. In fatigue failure, it is reasonable to obtain estimates of average dimensionless rate $\bar{V}_{j}=2 \bar{\Delta}_{j} / N_{j}$ of crack advance per one loading cycle for plain specimens of finite width at the appropriate loading mode (7) and (8)

$$
\bar{V}_{j}=\frac{\pi^{2} A^{2}}{4} \frac{\left(\bar{\varepsilon}_{\infty, j-1}\right)^{2+1 / C}}{\left(\bar{\varepsilon}_{p}\right)^{1 / C}}\left[Y\left(\bar{l}_{j-1} / \bar{L}\right) \bar{\sigma}_{\infty}\right]^{2} \bar{l}_{j-1},
$$

$j=1,2, \ldots, j^{*}-1$.

\section{Averaged rates of macro-crack tip advance}

Intsead of the discontinous function $\bar{V}_{j}$ in relation (9), we consider continous function $\bar{V}=\bar{V}(\bar{l})$, argument of which is continuously changing. The continuous function $\bar{V}=\bar{V}(\bar{l})$ and the discontinuous function $\bar{V}_{j}$ describe all three process stages: initiation, evolution, and completion of the process of macro-crack tip advance (load level $\bar{\sigma}_{\infty}=$ const, characteristic of material $\bar{\varepsilon}_{p}$, and specimen dimensions $\bar{L}$ are assumed to be given). Two-sided estimates of crack lengths, for which the Paris law is appropriate, have been obtained ( $\zeta$ is small positive number)

$$
\left[\left(1 / \bar{\sigma}_{\infty}-1\right) / \zeta\right]^{2} \leq 2 \bar{l} \leq 2 \bar{L}\left(1-\bar{\sigma}_{\infty}\right) .
$$

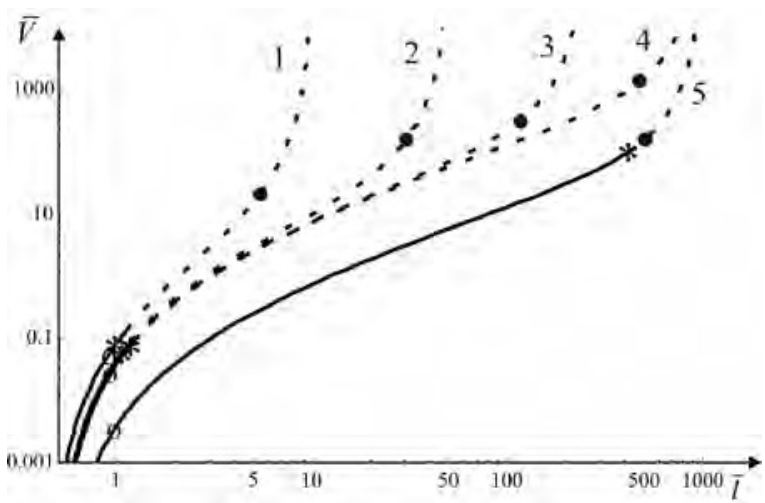

Fig. 3. The average rate of crack tip advance.

In Fig. 3, plots of averaged dimensionless rate of crack tip advance $\bar{V}=f\left(\bar{l}, \bar{\sigma}_{\infty}, \bar{\varepsilon}_{p}, \bar{L}, C\right)$ are given in log-log coordinates. Curves in Fig. 3 have been constructed via relation (9) for linear damage summation $C=1$ : when curves $1-4$ were plotted, the parameters $\bar{\sigma}_{\infty}=0.5, \bar{\varepsilon}_{p}=2$, and $\bar{L}=10,50,250$, and 1000 , respectively, were used; calculations for curve 5 were performed with parameters $\bar{\sigma}_{\infty}=0.5, \bar{\varepsilon}_{p}=20$, and $\bar{L}=1000$. In Fig. 3, light and dark dots on curves correspond to the left- and right-side inequalities in relation (10). Middle sections of curves are lengths of cracks for which the Paris law "works" well. To the right of asterisks depicted on curves $1-5$, failure of the specimen takes place in accordance with relation (4), therefore, the conditional sections of curves $1-5$ are continued as dotted lines. Curve 1 almost completely falls into the region of short macro-cracks. 


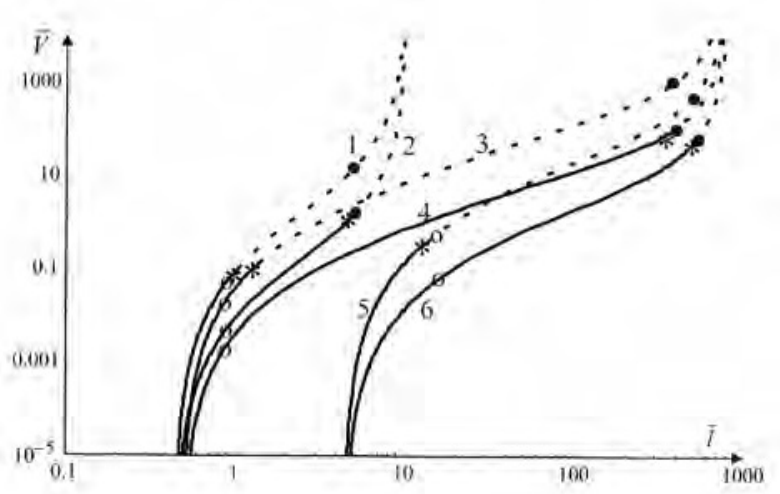

Fig. 4. The average rate of crack tip advance for linear damage summation.

Plots of averaged dimensionless rate of crack tip advance $\bar{V}=\bar{V}\left(\bar{l}, \bar{\sigma}_{\infty}, \bar{\varepsilon}_{p}, \bar{L}, C\right)$ in Fig. 4 are given in double logarithmic coordinates. Curves in Fig. 4 have been plotted via relation (9) taking into account the linear damage summation $C=1$ : the following parameters were used for construction of the curves: $\bar{\sigma}_{\infty}=0.5$ and $\bar{L}=10$ for curves 1 and $2, \bar{\sigma}_{\infty}=0.5$ and $\bar{L}=800$ for curves 3 and 4 , and $\bar{\sigma}_{\infty}=0.25$ and $\bar{L}=800$ for curves 5 and $6\left(\bar{\varepsilon}_{p}=2\right.$ and $\bar{\varepsilon}_{p}=20$ are relatuve margin of plastic strain of material for uneven and even curves, respectively, and $\mu=0)$. Light and dark dots on curves in Fig.4 correspond to the left- and right-side inequalities in relation (10). To the right of asterisks depicted on these curves, failure of the specimen takes place in accordance with relation (4), therefore, the conditional sections of the curves are continued as dotted lines. Each of these curves descrides initiation, evolution, and completion of the process of macro-crak tip advance. Middle sections of all the curves correspond to Paris' curves with some degree of certainty.

The exellent representation of all stages of the process is shown in monograph [6, p.27, Fig. 1.18]. In this mongraph and handbook [7], all three stages of the process and transition modes are discussed in detail. Howeever, the main attention of these authors $[6,7]$ is concentrated on the representation of these diagrams in the form of dependencies on SIF that does not agree with the results obtained for small-scale yielding in quasibrittle materials (9).

Given in Fig. 5 are curves characterizing the influence of relative plastic strain margin of material $\bar{\varepsilon}_{p}$

on the rate of crack tip advance $\bar{V}$. Curves $1-6$ have been plotted with account of linear damage summation $C=1$ for the parameters $\bar{\varepsilon}_{p}=1,2,5,10,20$, and 40 , respectively ( $\bar{\sigma}_{\infty}=0.25$, and $\bar{L}=800$ ). Light and dark dots on the curves in Fig, 5 correspond to the left- and right-side inequalities from relation (10). To the rigth of asteriks depicted on the curves, failure of the specimen takes place in accordance with relation (4), therefore, the conditional sections of these curves are continued as dotted lines.

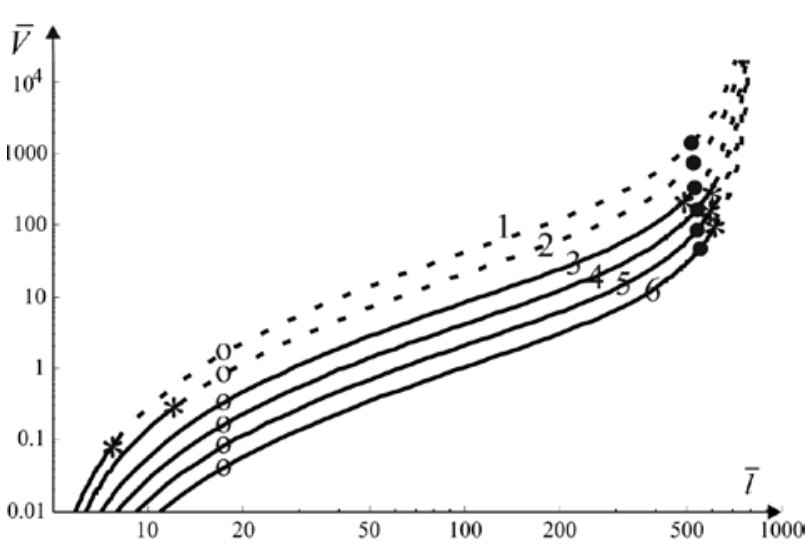

Fig. 5. The average rate of crack tip advance for various margin of plastic material strains $\bar{\varepsilon}_{p}$.

Plastic strain margin of material $\bar{\varepsilon}_{p}$ significantly affects the rates of crack tip advances $\bar{V}$. Let us consider two materials which differ only in margin of plastic strains of material $\bar{\varepsilon}_{p 0}$ and $\bar{\varepsilon}_{p 1}$. Then, using relation (9), the estimate can be written as

$$
\frac{\bar{V}_{0}\left(\bar{l}, \bar{\sigma}_{\infty}, \bar{\varepsilon}_{p 0}, \bar{L}, C\right)}{\bar{V}_{1}\left(\bar{l}, \bar{\sigma}_{\infty}, \bar{\varepsilon}_{p 1}, \bar{L}, C\right)} \approx\left(\frac{\bar{\varepsilon}_{p 1}}{\bar{\varepsilon}_{p 0}}\right)^{1 / C} .
$$

The margin of plastic strain after preliminary plastic deformation of material $\bar{\varepsilon}_{p 1}$ may be a small part of the plastic strain margin of basic material $\bar{\varepsilon}_{p 0}$, i.e., $\bar{\varepsilon}_{p 0} ? \bar{\varepsilon}_{p 1}$. Therefore, taking into account relation (11), the ratio of rates of crak tip advance $\bar{V}_{0} / \bar{V}_{1}$ for basic material and material after significant plastic deformation can reach from one to two orders of magnitude.

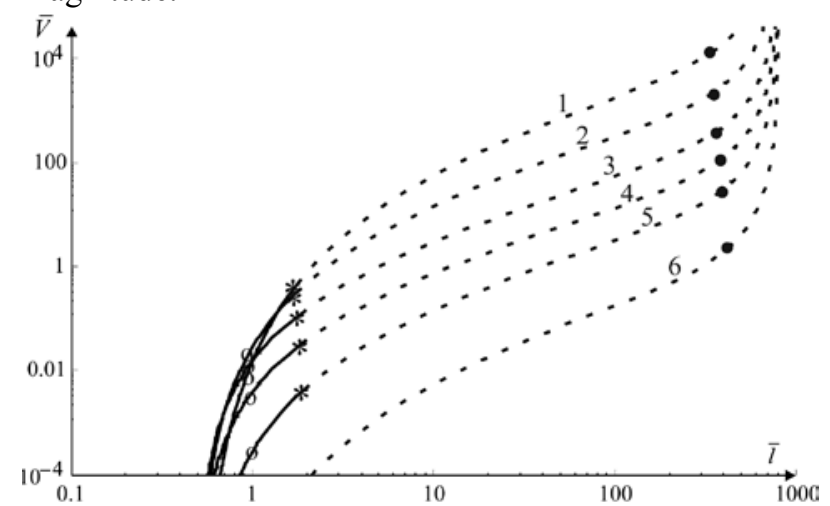

Fig. 6. Average rate of crack tip advances for for linear and nonlinear damage summation.

Given in Fig, 6 are curves reflecting effect of linear and nonlinear summation of materal damages on the averaged rate of crak tip advance $\bar{V}$. These curves have been plotted via relation (9) taking into account linear ( $C=1$ for curves 3 and 6 ) and nonlinear ( $C=0.25$ for curves 1 and 4 and $C=0.5$ for curves 2 and 5) damage summation. The parameters $\bar{\sigma}_{\infty}=0.5, \bar{L}=800$, and $\bar{\varepsilon}_{p}=2$ were used for construction the curves. The remaining notations are the same as those in Figs. $3-5$. If the conditional sections of these curves are excluded, 
then there are no sections on these curves, which can be treated as Paris' curves.

\section{The interrelation between the Coffin constant and characteristic rate of crack tip advance}

The proposed approximate relation (9) permits relate the Coffin constant with the charcteristic rate of crak tip advance drawing on their association at the second stage of crack tip advance when the Paris law "works" well. Let the characteristic rate $V_{0}=V_{0}\left(\bar{l}_{0}, \bar{\sigma}_{\infty 0}, \bar{\varepsilon}_{p 0}, \bar{L}_{0}, C\right)$ of crack tip advance be determined for some parametrs $\bar{l}_{0}, \bar{\sigma}_{\infty 0}, \bar{\varepsilon}_{p 0}, \bar{L}_{0}$ at the second stage of a process during laboratory experiment. The procedure for measurung this rate under laboratory conditins is well developed [8]. After simple transformations of relation (9), we obtain

$$
\begin{aligned}
& (1 / C)\left(\ln F_{0}-\ln A \bar{\varepsilon}_{p 0}\right)=\ln V_{0}^{\%}-\ln \left(\pi^{2} / 4\right)- \\
& -2 \ln F_{0}-2 \ln \left[Y\left(\bar{l}_{0} / \bar{L}_{0}\right) \bar{\sigma}_{\infty}\right]-\ln \bar{l}_{0} \\
& F_{0}=Y^{-1}\left(\frac{\bar{l}_{0}}{\bar{L}_{0}}\right)\left[\frac{1}{1-\bar{l}_{0} / \bar{L}_{0}}-\frac{1}{\bar{\sigma}_{\infty 0}}+Y\left(\frac{\bar{l}_{0}}{\bar{L}_{0}}\right) \sqrt{2 \bar{l}_{0}}\right] \sqrt{\frac{1}{2 \bar{l}_{0}}} .
\end{aligned}
$$

Relation (12) associates the constant $C$ and characteristic rate $\%$ based on the Paris law, i.e. $C=C(\%)$.

It is proposed to use in relation (9) the experimentaly determined $C$ constant from relation (12) for description of all three stages of crack tip advances including closely adjacent value of parameters $\bar{\sigma}_{\infty}$ and $\bar{L}$. It is to be recalled that derived relation (9) permits one to study behavior of cracks, lengths of which can be assignd just as to short, so to long cracks. Three stages of the process of crack tip advances are roughly estaimated by relations (10). Implementation of some or other stages of the process should be agreed with constrains imposed by the presence of two certain threshold values of crack lengths with the given loading intensity $\bar{\sigma}_{\infty}$. Relation (9) can be used for variable loading condititions. With a gradual decrease of loading intensity, the system can fall several times into the region, which corresponds to the region of short crack tip advance (10) (as compared with the behavior of curves 3 and 4 with that of curves 5 and 6 in Fig. 4).

\section{$6 S-N$ fracure diagrams}

The analysis of the crack tip advance does not fully satisfy the requirements of practice since, from a practical point of view, one has to know how many cycles are left before complete failure when the stress intensity of loading $\bar{\sigma}_{\infty}$ and the length of initial crack $2 \overline{l_{0}}$ are given. Suppose that this crack length $2 \overline{l_{0}}$ has been obtained at the latest inspection of the structure.
Let us study relation (9) in more detail. Intsead of the discontinous function $\bar{V}_{j}$ in relation (9), we consider continous function $\bar{V}=\bar{V}(\bar{l})$, argument of which is continuously changing. Assume that specimens of the infinite widh, i,e., $\bar{L}=\infty$, are under consideration. Let the right-hand side of relation (9) be a continuos function. The left-side of relation (9) can be represented as $\bar{V} \approx d 2 \bar{l} / d N$, if we assume that $N$ is the continous function. Then relation (9) is approximately rewritten in thr form of a differential equation

$$
\begin{gathered}
d 2 \bar{l} / d N \approx f(2 \bar{l}), \\
f(2 \bar{l}) \approx \frac{\pi^{2}}{8\left(A \bar{\varepsilon}_{p}\right)^{1 / C}}\left[1+\left(1-\frac{1}{\bar{\sigma}_{\infty}}\right) \sqrt{\frac{1}{2 \bar{l}}}\right]^{2+1 / C} \bar{\sigma}_{\infty}^{2} 2 \bar{l} .
\end{gathered}
$$

This ordinary differential equation (13) may be integrated

$$
\begin{gathered}
N^{*} \approx \int_{2 \bar{l}_{0}}^{2 \bar{l}^{(2)}} f^{-1}(2 \bar{l}) d 2 \bar{l}+b, \\
\bar{\sigma}_{\infty} \approx\left(1+\sqrt{2 \bar{l}^{(1)}}\right)^{-1}, \bar{\sigma}_{\infty} \approx\left[1+\left(1-A \bar{\varepsilon}_{p}\right) \sqrt{2 \bar{l}^{(2)}}\right]^{-1},
\end{gathered}
$$

where $N^{*}$ is the critical number of cycles before failure, $b$ is the constant of integration, $2 \bar{l}_{0}$ is the initial crack length satisfying the constrains $2 \bar{l}^{(1)}<2 \bar{l}_{0}<2 \bar{l}^{(2)}$, and $2 \bar{l}^{(2)}$ is the critical crack length. This critical crack length $2 \bar{l}^{(2)}$ is the second threshold value of a crack length at the given loading level $\bar{\sigma}_{\infty}<1$ of quasibrittle material. Thus, both threshold values of crack lengths $\bar{l}^{(1)}$ and $\bar{l}^{(2)}$ in relation (14) are used at the given loading level $\bar{\sigma}_{\infty}$ with account of constrains,. The constant $b$ is chosen from the condition: for $2 \overline{l_{0}}=2 \bar{l}^{(2)}$, the critical number of cycles before failure $N^{*}=1$, i.e., $b=1$, that corresponds to monotonic loading. Approximate relation (14) is one again approximate alternative of the damage tolerant criterion.

Integral (14) is an improper one since $2 \bar{\Delta} \rightarrow 0$ for $2 \overline{l_{0}} \rightarrow 2 \bar{l}^{(1)}$. This integral permits representation in the closed form when the parameter $2+1 / C$ is the the integer value, i.e., e. $2+1 / C=3,4,5,6$, and $2+1 / C=7$.

Given in Figs. 7 and 8 are the $N-l_{0}$ fracture diagrams, which are estimates of lifetime for specimens with an initial crack at the given loading level $\bar{\sigma}_{\infty}$. Curves 1, 2, and 3 shown in Fig. 7 are plotted via relation (14) for the loading intensities $\bar{\sigma}_{\infty}=0.55 ; 0.5$, and $\bar{\sigma}_{\infty}=0.4$ on linear damage summation $C=1$ $\left(\bar{\varepsilon}_{p}=5, \mu=0.33\right)$. Points marked with asterisks indicate the places where a specimen is finally broken down. Thick segements on the basic curves reflect the selected complex three-level loading mode (load range shiftings are labeled by thin dotted lines). Due to change 
of the loading level, such modes of fatigue fracture are practible when the system can fall several times into areas of short crack lengths.

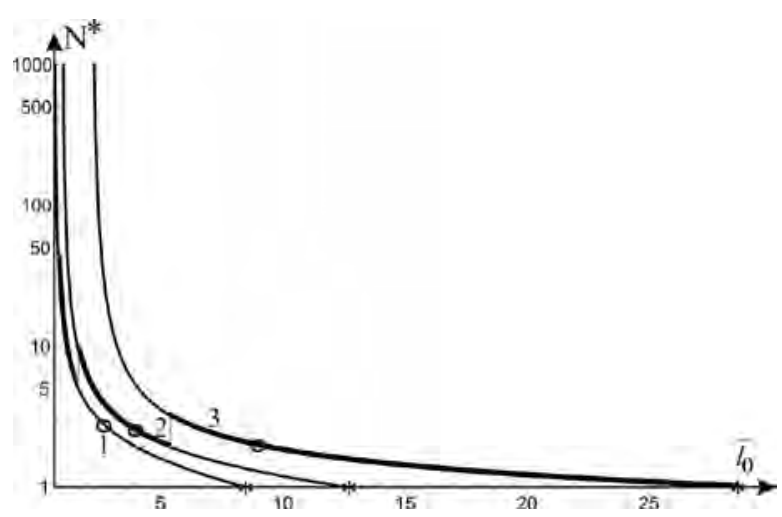

Fig. 7. $N-l_{0}$ fracture diagrams.

Curves 1, 2, and 3 in Fig. 8 have been plotted via relation (14) for low loading intensities $\bar{\sigma}_{\infty}=0.2 ; 0.1$, and $\bar{\sigma}_{\infty}=0.05$ on linear damage summation $C=1$ $\left(\bar{\varepsilon}_{p}=5, \mu=0.33\right)$. Points marked with asterisks indicate the places where a specimen is finally broken down. At low loading intensities $\bar{\sigma}_{\infty}$, such modes are specific in which an intial crack does not grow.

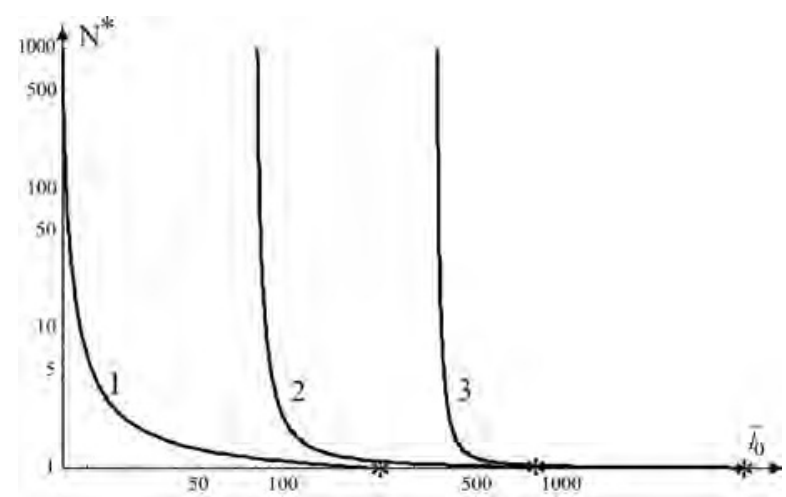

Fig. 8. $N-l_{0}$ fracture diagrams for different loading intensities.

If $S-N$ or $N-l_{0}$ fracture diagrams and the crack growth rate $\bar{V}$ are taken into account, then it will be possible to estimate transition from relatively secure operating conditions of a structure with a crack to dangerous loading mode.

\section{Conclusion}

Step-wise crack propagation in quasi-brittle materials under cyclic loading conditions has been considered. For a model of deformable body, the model of elastic ideally-plastic material having the ultimate tensile strain is taken. When basic material undergoes nonlinear deformation, the material is embrittled.

For analysis of the process mentioned above, it is proposed to use modified diagrams of quasi-brittle fracture of deformable bodies. Reference diagrams have been plotted within the context of quasi-linear fracture mecanics when the small-scale material yelding is realized in the vicinity of a crack tip and the singularity of the stress field at the tip of a crak is partally retained.

Two theshold values of critical parameters are proposed for description of the process of step-wise crack tip advance in responce to fatigue loading modes. Both theshold values are given in the explicit form including that for short macro-cracks. The second theshold value is a variable and it is directly associated with material damage in a pre-fracture zone. Under nonlinear deformation of material in the pre-fracture zone, the plasticity margin is spent due to material embrittlement during each loading cycle.

Constants in the described model are proposed to be selected for individual material from the data obtained during three laboratory experiments based on $i$ ) approximation of a real diagram stress versus strain, ii) searching critical SIF, and iii) Coffin's constants or the characteristic rate of crack tip advance for the second process stage.

The analysis of all three process stages of crack tip afvances and their dependence on geomentric parameters of cracks and specimens, characterisics of material, and intensity of fatigue loading under pulstaing loading appliance has been performed in detail. For the averaged process, crude rwo-sided estimates for transition from the first to the second stage and from the second to the third stage have been pointed out, fairly simple analitical expressions for describing the whole process, including the case when some stages are absent have been derived. The plotted $N-S-\bar{l}_{0}$ diagrams charcterize lifetime of specimens having initial defects. All the constructions have peformed in terms of cracks. There is the fracture mechanics approach, which specifically treats growing cracks by the methods of fracture mechanics.

This work was supported by the Russian Foundation for Basic Research (Grant No. 16-08-00483).

\section{References}

1. V.M. Kornev, Physical. Mesomech., 14, 5, pp. 3145 (2011) (in Russian)

2. V.M. Kornev, Physical. Mesomech., 19, 2, pp. 8099 (2016) (in Russian)

3. H. Neuber, Kerbspannungslehre: Grunglagen fur Genaue Spannungsrechnung (1937)

4. V.V. Novozhilov, Prikl. Mat. Mekh., 33, 2, pp. 212222 (1969) (in Russian)

5. V.M. Kornev, Engin. failure analysis, 35 (2013), pp. 533-544

6. V.V. Bolotin, Mechanics of fatigue (1998)

7. O.N. Romaniv, S.Ya. Yarema, G.N. Nikiforchin, N.A. Makhutov, M.M. Stadnik, Fracture Mechanics and strength of materials, Vol. 4 (1990)

8. R.O.Ritchie, Intern. J. Fracture. 100, pp 55-83 (1999) 